\title{
EVALUATING COMPUTER PROGRAM \\ PERFORMANCE ON THE CRAY-1
}

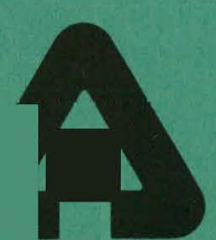

UOAC-AUA. USDOE

ARGONNE NATIONAL LABORATORY, ARGONNE, ILLINOIS Prepared for the U. S. DEPARTMENT OF ENERGY under Contract W-31-109-Eng-38

\author{
by \\ Larry Rudsinski \\ with \\ Gail W. Pieper

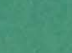




\section{DISCLAIMER}

This report was prepared as an account of work sponsored by an agency of the United States Government. Neither the United States Government nor any agency Thereof, nor any of their employees, makes any warranty, express or implied, or assumes any legal liability or responsibility for the accuracy, completeness, or usefulness of any information, apparatus, product, or process disclosed, or represents that its use would not infringe privately owned rights. Reference herein to any specific commercial product, process, or service by trade name, trademark, manufacturer, or otherwise does not necessarily constitute or imply its endorsement, recommendation, or favoring by the United States Government or any agency thereof. The views and opinions of authors expressed herein do not necessarily state or reflect those of the United States Government or any agency thereof. 


\section{DISCLAIMER}

Portions of this document may be illegible in electronic image products. Images are produced from the best available original document. 
The facilities of Argonne National Laboratory are owned by the United States Government. Under the terms of a contract (W-31-109-Eng-38) between the U. S. Department of Energy, Argonne Universities Association and The University of Chicago, the University employs the staff and operates the Laboratory in accordance with policies and programs formulated, approved and reviewed by the Association.

\section{MEMBERS OF ARGONNE UNIVERSITIES ASSOCIATION}

The University of Arizona

Carnegie-Mellon University

Case Western Reserve University

The University of Chicago

University of Cincinnati

Illinois Institute of Technology

University of Illinois

Indiana University

Iowa State University

The University of Iowa
Kansas State University The University of Kansas Loyola University Marquette University Michigan State University The University of Michigan University of Minnesota University of Missouri Northwestern University University of Notre Dame
The Ohio State University Ohio University

The Pennsylvania State University Purdue University

Saint Louis University

Southern Illinois University The University of Texas at Austin Washington University

Wayne State University

The University of Wisconsin

NOTICE

This report was prepared as an account of work sponsored by the United States Government. Neither the United States nor the United States Department of Energy, nor any of their employees, nor any of their contractors, subcontractors, or their employees, makes any warranty, express or implied, or assumes any legal liability or responsibility for the accuracy, completeness or usefulness of any information, apparatus, product or process disclosed, or represents that its use would not infringe privately-owned rights. Mention of commercial products, their manufacturers, or their suppliers in this publication does not imply or connote approval or disapproval of the product by Argonne National Laboratory or the U. S. Department of Energy.

Printed in the United States of America Available from

National Technical Information Service

U. S. Department of Commerce 5285 Port Royal Road

Springfield, Virginia 22161

Price: Printed Copy $\$ 4.50$; Microfiche $\$ 3.00$ 


\section{EVALUATING COMPUTER PROGRAM PERFORMANCE ON THE CRAY-1 \\ by}

Larry Rudsinski

with

Gail w. Pieper

Applied Mathematics Division

malor, melior

This report was prepared as an account of work This the United States nor the United States Department of Energy, nor any of their employees, nor sny of their Energe, nor any of their on theis employees, makes coniractors, subcontracton, or her er assumes any legal any wartanty. express or torled, or ass liability or responsibily for the accuracy. completeness or usefulness of any intormation, apparalus, product or process disclosed, or represents that its use would not infringe privately owned rights.

January 1979 


\section{THIS PAGE \\ WAS INTENTIONALLY \\ LEFT BLANK}




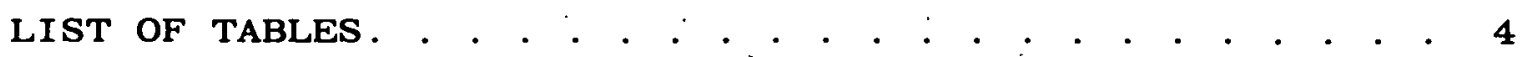
ABSTRACT. . . . . . . . . . . . . . . . . . . . . . . . . 5

I . INTRODUCTION . . . . . . . . . . . . . . . . 6

I I . MACHINE SELECTION. . . . . . . . . . . . . . . . . 7

III . PERFORMANCE COMPARISONS: CRAY-1 AND IBM 370/195. 8

A. Ptolemy (Physics). . . . . . . . . . . . 8

B. Dirac-slater (Solid State Sciences). . . . . . 9

C. PIC (Chemistry). . . . . . . . . . . . . . . 9

1. PARMOVE (Chemistry/Applied Mathematics). - 9

2. VFFA and VFFS (Chemistry/Applied

Mathematics). . . . . . . . . . . . . . 10

D. MD (Solid State Sciences). . . . . . . . . 10

E. DIF3D (Applied Physics). . . . . . . . . . . . 11

F. SAS (Reactor Analysis and Safety). . . . . . . 11

G. MATEQ (Reactor Analysis and Safety). . . . . . 12

H. JWMBENCH (H1gh Energy Physics). . . . . . . . 13

IV. PERFORMANCE COMPARISONS: CFT SCALAR, CFT VECTOR, AND OPTIMUM CRAY VECTOR CODE. . . . . . . . . . . . 14

V. MACHINE ENVIRONMENT STUDIES. . . . . . . . . . . . 18

VI. I IO TESTS. . . . . . .

VII . DISCUSSION . . . . . . . . . . . . . . . . . . . . . . 20

VIII . SUMMARY AND CONCLUSIONS . . . . . . . . . . . . . . 22

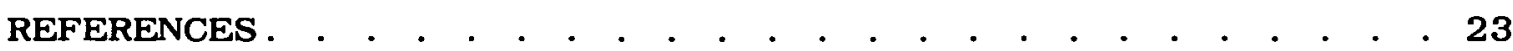

ACKNOWLEDGMENTS . . . . . . . . . . . . . . . . . . . . . . . . . 24

APPENDIX A. . . . . . . . . . . . . . . . . . . . . . . . 25 


\section{LIST OF TABLES}

No. $\quad$ Title

$\underline{\text { Page }}$

I. Timing Performance (in microseconds per particle, $64^{2}$ particles $/ 64 \times 64$ mesh) for PIC. . ... . . . . . 9

II. Timing Performances (in microseconds per particle) for PARMOVE. . . . . . . . . . . . . . . . . . . . 9

III. Timing Performance (in ms) for VFFA and VFFS Together on a $64 \times 64$ set of Data Points. . . . . . 10

IV. Performance Comparisons of Versions of the MD Code (in seconds per time step, 500-particle system). . 10

V. Comparison of Running Times (in seconds) for SAS Routines. . . . . . . . . . . . . . . . . . . 12

VI. Execution Times (in seconds) Using ICEPEL before and after Modifications. . . . . . . . . . . . . . 12

VII. Execution Times (in ms) Using JWMBENCH with and without Modifications. . . . . . . . . . . . . . . . 13

VIIIa. SUM $=$ SUM $+A(I)$ (all timings in microsecond). . . 15 VIIIb. Comparisons of various Versions by Ratios. . . . 15

IXa. Example 2 (time in microseconds), , . , , . . . . . 16 IXb. Example 2 (ratios). . . . . . . . . . . . . . . 16

X. Size of PARMOVE (in decimal-words). . . . . . . . . . 17

XI . Fourier Analysis Routines VFFA and VFFS (times in ms). . . . . . . . . . . . . . . . . . . . . . . 17

XII. CRAY-1 I/O Test Results. . . . . . . . . . . . . . 19

XIII. Improvement Factor on the CRAY-1 over the
IBM $370 / 195$ with Various Versions of Code. . . . 20 


\title{
EVALUATING COMPUTER PROGRAM PERFORMANCE ON THE CRAY-1
}

\author{
by \\ Larry Rudsinski \\ with
}

Gail W. Pieper

\begin{abstract}
The Advanced Scientific Computers Project of Argonne's Applied Mathematics Division has two objectives: to evaluate supercomputers and to determine their effect on Argonne's computing workload. Initial efforts have focused on the CRAY-1, which is the only advanced computer currently available. Under a grant from the National Center for Atmospheric Research, users from seven Argonne divisions executed test programs on the CRAY and made performance comparisons with the IBM 370/195 at Argonne. This report describes these experiences and discusses various techniques for improving run times on the CRAY.

Direct translations of codé from scalar to vector processor reduced running times as much as two-fold, and this reduction will become more pronounced as the CRAY compiler is developed. Further improvement (two- to ten-fold) was realized by making minor code changes to facilitate compiler recognition of the parallel and vector structure within the programs. Finally, extensive rewriting of the FORTRAN code structure reduced execution times dramatically, in three cases by a factor of more than 20; and even greater reduction should be possible by changing algorithms within a production code.

We conclude that the CRAY-1 would be of great benefit to Argonne researchers. Existing codes could be modified with relative ease to run significantly faster than on the $370 / 195$. More important, the CRAY would permit scientists to investigate complex problems currently deemed infeasible on traditional scalar machines. Finally, an interface between the CRAY-1 and IBM computers such as the $370 / 195$, scheduled by Cray Research for the first quarter of 1979, would considerably facilitate the task of integrating the CRAY into Argonne's Central Computing Facility.
\end{abstract}




\section{I . INTRODUCTION}

Argonne National Laboratory carries out fundamental research in the physical. biomedical; and environmental sciences and serves as a major center for energy research and development. Essential to these activities is the availability of powerful computing facilities to carry out data analyses and modeling of physical systems.

Motivated by this need, by the recommendation of a consultant to Argonne, 1 and by recent advances in large-scale scientific computers, the Applied Mathematics Division (AMD) initiated the Advanced Scientific Computers Project to evaluate supercomputers and to determine their potential impact on Argonne's workload.

User involvement is essential to the goals of the project. This involvement can be broken down into two stages:

a. classes to familiarize the user with various advanced machines and with programming techniques needed to take advantage of the machines ' architecture, and

b. execution of programs on the machines to enable the user to evaluate the performance of his code.

Network access for this project is also essential to maintain user interest and effectiveness. We feel that several job turnarounds are needed for each user to become familiar with the machine under study. Moreover, after the user develops and executes programs that are representative of his workload, he will want to compare the performance of these programs on the supercomputer and on machines he is currently using. We therefore requested and received approval for network access, using AMD's Remote Job Entry station. 


\section{MACHINE SELECTION}

The CRAY-1 was chosen to begin our investigation because it is the only machine currently available that qualifies as an advanced scientific computer, or Class VI (one capable of executing 20 to $60 \mathrm{million}$ floating point operations per second, or 20-60 MFLOPS). Other computers (namely, the Star 100A and the Burroughs Scientific Computer) are expected to qualify as Class VI machines in late fall of 1978 or spring of 1979 , at which time we plan to examine them.

Initially, the CRAY at Los Alamos Scientific Laboratory (LASL) seemed a good possibility, since it is available on request to other national laboratories. Unfortunately, to gain direct access, one needs to be on site and to have a $Q$ clearance. It is possible to use LASL's open CDC 6600 with the CRAY compiler on it, and to develop the programs from Argonne, but someone would still have to go to LASL to execute the programs on the CRAY itself.

An alternate possibility of getting time on the CRAY-1 at the National Center for Atmospheric Research (NCAR) at Boulder, Colorado, was subsequently investigated. At NCAR, computer time is not purchased; instead, the computer resources are allocated to various projects as grants. After discussions with Jeanne Adams, the liaison between the Laboratory and NCAR, we received approval to conduct performance comparisons with the $370 / 195$ and to compare CRAY scalar and optimized vector code. Two hours of CRAY-1 time was granted, as well as CDC 7600 CRUs allocation.

This latter allocation is necessary because NCAR front-ends its CRAY-1 computer with a CDC 7600. As the hub of the NCAR configuration, the 7600 is also connected to the MODCOMP II, which serves as a Remote Job Entry station, and to, the Ampex terabit memory mass storage unit. When a job is sent from Argonne to NCAR, the MODCOMP II receives the file and then passes it on to the 7600 , which in turn sends it to the CRAY for processing.

Only a few days were needed to get the project number and accounting into NCAR's system. The Argonne offsite computing station, which is operated by AMD, already was able to handle the UT200 protocol needed to communicate to NCAR as a Remote Job Entry terminal, and only minor changes were needed in the character translate table to handle special characters required for sending CRAY Assembly Language (CAL).

The GSA approval for using commercial telephone lines to connect Argonne and NCAR was received within four months. 


\section{PERFORMANCE COMPARISONS: CRAY - 1 AND IBM 370/195}

Four classes were given at Argonne on the CRAY-1 in May 1978; thiry-two users participated. Discussions focused on methods for translating code and on minor changes in vector techniques to take advantage of the CRAY architecture.

A few problems were discovered, including errors in the compiler documentation, which led to misunderstanding about exponent range, and discrepancies between the floating-point arithmetic unit and the machine documentation (see below, Sec. V).

Nevertheless, reaction to the system was predominantly favorable: The effort established the relative ease with which Argonne scalar codes could be run on the CRAY. Additionally, comparison runs with the IBM 370/195 indicated that significant reduction in execution time could be achieved with the supercomputer.

In the tables that follow, we have headed two columns as traditional and rewritten. Traditional denotes programs that were executed with only those changes to the FURTRAN that were needed to eliminate FORTRAN errors and to obtain correct results. Rewritten denotes the same code but with modules rewritten so that the CRAY FORTRAN (CFT) compiler would generate vector instructions rather than scalar instructions. Rewritten does not in any way imply that the whole code was modified; in fact, in most of the programs, only a very small section was modified to satisfy the CFT compiler criteria for vectorization. No effort was made to improve the run time efficiency of any of the test codes for the IBM $370 / 195$ other than those cases specifically stated. In column 3 of Table XIII, we have denoted by an asterisk those codes known to be efficiently written for the $370 / 195$.

It should be noted, however, that in a few cases the code modifications needed to generate efficient code for one machine, the CRAY-1, did quite the contrary on another, the 370/195. In Tables I-III, the changes made in the rewritten code appear to have destroyed the positive effects of the IBM cache memory, increased the number of subscript calculations, and forced more variables to be stored through the cache.

We also note that care should be taken in comparing timing results obtained from code generated by FORTRAN H extended opt 2 and by the CFT compiler. The CFT compiler, which is used at NCAR along with the standard supported CRAY-1 operating systcm, is not so sophisticated as the FORTRAN $H$ extended opt 2 . Scalar instruction scheduling is currently being improved, and these improvements should be available in the next version of the CFT.

The following examples are representative of user experience.

\section{A. Ptolemy (Physics)}

One of the main computational sections of Ptolemy (a program for computing nuclear elastic and direct-relation cross-sections) was extracted and executed on the CRAY-1. After a few debug runs, the 5000-line section gave answers in agreement with the IBM $370 / 195$ results.

Initially the CRAY-1 version executed only slightly faster than the $370 / 195$ version. However, after analyzing the inner loop of the calculation, we found an overflow test that was unncccssary in view of the increased exponent range of the CRAY. Removal of the test and accompanying code improved execution time by a factor of two over the $370 / 195$.

Subsequently, more extensive rewriting of this section of Ptolemy was undertaken. Rather than calculating one value per pass, the inner loop was inverted to permit many simultaneous calculations by the CRAY compiler. Overall performance was a factor of ten faster on the CRAY-1 than on the IBM 370/195. 


\section{B. Dirac-slater (Solid State Sciences)}

A relativistic atomic structure code was selected which uses a majority of its CPU cycles to solve one-dimensional coupled differential equations. The code involves a predictor-corrector scheme in which the initial prediction depends upon the solution from the previous iteration before the correction(s) can be accomplished. We ran a $\mathrm{U}^{+++}$ion problem and compared performances on the CRAY and on the 370/195. With simple code translation involving very few vector loops, the CRAY ran 2.26 times faster. Some minor changes, namely, pulling part of the radial calculation out where it could be converted to vector form, produced an additional 15 percent gain in execution time such that the CRAY was running 2.6 times faster than the $370 / 195$. In both cases the calculations agreed with the same problem executed on the IBM $370 / 195$.

\section{PIC (Chemistry)}

Particle-in-cell trajectory $(P I C)^{2}, 3$ codes are useful in testing approximate models of ion-ion plasma in an electrical quadrupole mass filter. Since such tests require a prohibitive amount of time on the $370 / 195$, runs on the CRAY were considered. Instead of a direct translation of an Argonne PIC code, we chose to utilize the major compute loops of a PIC code developed at LASL, which is more efficient on the $370 / 195$ (a factor of 18) than the existing ANL version and which makes more use of vector techniques and has reduced timings on the CRAY.

Accordingly, a new front-and back-end was written for the LASL codes and adapted to the specific model. The combined program was then tested on both the IBM $370 / 195$ and the CRAY-1. The results are shown in Table I.

TABLE I

Timing Performance (in microseconds per particle, $64^{2}$ particles/64X64 mesh) for PIC

$\begin{array}{lccc}\text { Machine } & \text { Traditional } & \text { Rewritten } \\ 370 / 195 & 43 & \\ \text { CRAY-1 } & 21 & 82 \\ \text { nnn } & & 8\end{array}$

\section{PARMOVE (Chemistry/Applied Mathematics)}

One PIC routine, PARMOVE (which updates the particle positions and velocities in a given time step and consumes approximately 85 percent of the cude's execution time), was run on the ckaY-1 and on the $1 B M 370 / 195$.

Table II shows the timing performances for various versions of PARMOVE.

TABLE II

Timing Performances (in microseconds per particle) for PARMOVE

Mach ine

$370 / 195$

CRAY-1 (CFT)

\section{Tradit ional} 33 18
Rewritten
46

6 
It should be noted that better performance was obtained for both the scalar and vector version of PARMOVE compiled on FTN. "FTN is the current compiler supported by Control Data Corporation for their CYBER series computers. LASL has taken this compiler and modified the code generator to generate object code for the CRAY-1. Again, as CFT matures and later versions improve the instruction scheduling, the performance should improve to at least the same quality as the FTN compiler.

\section{VFFA and VFFs (Chemistry/Applied Mathematics)}

Also part of the PIC codes are two FORTRAN routines, VFFA and VFFS, which perform Fourier analysis and Fourier synthesis on many points at once. These two routines make up approximately two-thirds of the calculations in solving the Poisson or Helmholtz equation using the direct method. ${ }^{6}, 7$

Table III compares the times for VFFA and VFFS together on the $370 / 195$ and the CRAY-1.

TABLE I I I

Timing Pęrformance (in ms) for VFFA and VFFS Together on a $64 \times 64$ set of Data Points

$\begin{array}{lccc}\text { Machine } & \text { Traditional } & \text { Rewritten } \\ 370 / 195 & 47 & 91 \\ \text { CRAY-1 } & 21 & 3\end{array}$

\section{MD (Solid State Sciences)}

Molecular Dynamics (MD) codes, used for studying 500-particle systems, are similar to the PIC codes described in Sec. III.C. PIC simulation programs are generally used to study physical phenomena starting at a non-equilibrium state and following the problem until an equilibrium state is reached. MD programs, on the other hand, are used to study dynamic equilibrium; that is, the physical state of the system is at equilibrium at the start, and it stays in an equilibrium state throughout the calculation. MD codes also do not use a background stationary mesh to influence the motion and position of the particles: instead, each particle directly influences the motion and the position of every other particle in an infinite system generated by standard periodic boundary conditions.

We tested an 800-card version on both the CRAY-1 and the 370/195. Each code executed the same problem in the MD code for 150 time steps. The 370/195 required 90 seconds (i.e., $0.6 \mathrm{sec} / \mathrm{time}$ step), and the CRAY-1 required 64 seconds (i.e., $0.43 \mathrm{sec} / \mathrm{time}$ step). Table IV gives the performance comparisons.

\section{TABLE IV}

Performance Comparisons of Versions of the MD Code (in seconds per time step. 500-particle system)

$\begin{array}{lccc}\text { Machine } & \text { Traditional } & \text { Rewritten } \\ 370 / 195 & 0.60 & \\ \text { CRAY-1 } & 0.43 & & 0.36\end{array}$


The current CFT compiler was unable to vectorize any of the FORTRAN in the major calculation sections of $M D$ in its present form. By timing various sections of MD, however, we determined that a small section of code required 10 out of the 64 seconds to execute. Rewriting the section in a more vectorized FORTRAN, using several calls to a subroutine SUM (see Sec. IV), correcting IF tests to function calls, and calling the vector version of SIN and COS, we reduced the time in this section of code from 10 seconds to 0.37 seconds. The total execution time on the CRAY-1 was reduced to $53.5 \mathrm{sec} / 150$ time steps, or $0.36 \mathrm{sec} / \mathrm{t}$ ime step.

MD still has a section of code where particle quantities are updated for a given time step. This section, like PARMOVE in the PIC codes, consumes a major portion of the execution time. Close analysis indicates that the section, again like PARMOVE, can be rewritten so that the CFT compiler can generate vector code. This effort is beyond the scope of our current project; it is, however, being considered by the Solid State Sciences Division, which is interested in MD calculations related in general to the theory of disordered systems (e.g., liquids) and in particular to the study of particle motions in ionic conductors (related to problems arising in the study of battery materials).

\section{E. DIF3D (Applied Physics)}

DIF3D is a fast reactor finite-difference theory code. ${ }^{\circ}$ The code has two compute sections, an outer fission source iteration and a within-group inner iteration. The inner iteration consumes 60\%-70\% of execution time for the total program; hence, it is this part of the calculation that was executed on the CRAY-1.

The inner iteration uses the Successive Line Overrelaxation (SLOR) method with optimized SLOR factors to solve Helmholtz's equation on a two- and threedimensional mesh depending on the problem. A line gives rise to a tridiagonal matrix equation ( $A x=y$ ) that is solved for $x$ by using the $L-U$ decomposition. This procedure requires a substantial number of recursive operations that do not lend themselves to vectorization.

Thus, the inner iteration executes with speed comparable to the IBM 370/195. Vectorization of the non-recursive codes in the L-U decomposition, at the expense of extra memory. reduced the execution time by 30 percent, i.e., 1.30 times faster on the CRAY-1 than on the 370/195. To obtain better results, we are currently exploring techniques for solving both the two- and three-dimensional Helmholtz equation that are more conducive to vector and parallel computers.

\section{F. SAS (Reactor Analysis and Safety)}

SAS codes are used to analyze the consequences of hypothetical accidents in fast breeder reactors. Because these codes account for 5-10 percent of the total Argonne computing load, and because SAS applications studies are being constrained by limitations in computer speed, scientists in the Reactor Analysis and Safety Division were interested in determining whether the CRAY would be significantly faster than the IBM 370/195. Since timing the entire SAS3D code ( 40,000 cards) on the CRAY-1 was beyond the scope of this project, two typical modules from the SAS family were independently timed. First, a stand-alone version of one of the older SAS3A modules, previously used for timing studies on other computers, was run on the CRAY-1. The section timed included the TSHTR subroutine and the auxiliary routines that it calls to compute transient temperatures. Second, a section of the SAS4A code currently being written was run. This section involved the subroutine ALPHFN, which computes the density and thermal expansion coefficient of sodium as a function of temperature. The results of these timing studies are shown in Table $v$. 
TABLE $\mathrm{V}$

Comparison of Running Times (in seconds) for SAS Routines

IBM $370 / 195$

TSHTR (SAS3A) -

10 steps

ALPHFN (SAS4A) -

1000 calls
0.261
0.139
0.460
0.015

CRAY - 1

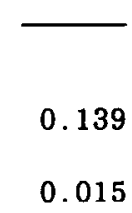

The CRAY-1 version of TSHTR, which generated very few vector instructions when compiled by CFT, ran almost twice as fast as the IBM $370 / 195$ version. It should be noted, however, that comparable performance was obtained on the CDC 7600. Again, as the current CRAY FORTRAN compiler matures, the CRAY-1 should become two to two and one-half times as fast as the CDC 7600 in executing scalar-generated code.

The SAS4A version of ALPHFN, which was completely vectorized by CFT, ran very well on the CRAY, sustaining an average rate of 51 MFLOPS. The CRAY-1 version executed a factor of 30 times faster than the $370 / 195$ version. The IBM $370 / 195$ performance, on the other hand, was unexpectedly poor. Breaking the one large DO loop (the main part of ALPHFN) into a number of smaller ones to take advantage of the $370 / 195$ loop mode might improve performance. However, achieving vector mode on the CRAY-1- requires considerably less effort by the programmer.

\section{G. MATEQ (Reactor Analysis and Safety)}

A program ICEPEL (used to perform two-dimensional analyses of piping systems) was translated and run on the IBM 370/195 and the CRAY-1. Performance time on the CRAY was a 1.7 improvement over the conventional machine.

Examining the program in more detail, we determined that 75 percent of the execution time was spent in a routine called MATEQ; developed at AMD for the numerical solution of least squares problems. MATEQ operates on both the matrix and the right-hand side each time it is called. Since the problem under study involved a fixed matrix with only the right-hand side changing, much unnecessary work was being performed. In addition, more accurate algorithms had become available since MATEQ was last revised in 1968-69. These new algorithms were implemented in LINPACK. 9

Two routines from the LINPACK package were used: DQRDC computes an orthogonal-triangular factorization of the matrix, and DQRSL solves the overdetermined linear system for any right-hand side. With the LINPACK replacements, the program executed 3.5 times faster on the $370 / 195$ and 4.4 times faster on the CRAY, giving an improvement factor of 2.2 on the CRAY compared to the $370 / 195$ (see Table VI).

TABLE VI

Execution Times (in seconds) Using ICEPEL before and after Modifications

IBM $370 / 195$

\begin{tabular}{lcc} 
& IBM 370/195 & CRAY-1 \\
\cline { 2 - 2 } & & \\
MATEQ & 134.5 & 79.8 \\
LINPACK Replacements & 40.7 & 18.3
\end{tabular}




\section{H. JWMBENCH (High Energy Physics)}

We also experimented with a program called JWMBENCH, which is designed to obtain execution timings for various machines. Two of IBM's Scientific Subroutine Package subroutines were used, MINV to invert the $15 \times 15$ matrix and GMPRD to multiply the inverse by the original matrix.

Initially the program took approximately $16.2 \mathrm{~ms}$ to run on the IBM $370 / 195$ and $1.5 .7 \mathrm{~ms}$ on the CRAY-1. Rewriting GMPRD to generate vector code significantly reduced program running time on the CRAY (down by $20 \%$ to $12.66 \mathrm{~ms}$ ). Interesting here is the fact that the vector techniques also improved the efficiency on the 370/195: the run time was $16.0 \mathrm{~ms}$.

This timing information must, however, be used with caution in comparing the power of the two computers. To illustrate, when we replaced the MINV algorithm with newer and more efficient techniques implemented in LINPACK, we obtained a dramatic improvement on the CRAY-1 compared to that on the $370 / 195$ (400\% vs 17\%). Table VII gives the times for various cases of JWMBENCH and illustrates just how much the CRAY-1 would have been underrated, had we not changed to better algorithms.

\section{TABLE VII}

Execution Times (in ms) Using JWMBENCH with and without Modifications

Original GMPRD Vectorized GMPRD LINPACK Replacement of MINV
16.2

16.0

15.7

13.3
12.6

3.0 


\section{PERFORMANCE COMPARISONS: CFT SCALAR, CFT VECTOR, AND OPTIMUM CRAY VECTOR CODE}

Section III illustrates the performance gains of the CRAY-1 over the IBM $370 / 195$ with varying degrees of code modification. It is also important to get some idea of the performance level of the software that currently exists. As stated in Section III, the CFT compiler is new and not so sophisticated as the IBM FORTRAN H opt 2 or CDC FTN opt 2 . Therefore, to better show how much more performance is available by rewriting in CAL, we ran four experiments comparing CFT scalar, CFT vector, and optimum vector performance.

The first example sums up a vector. Written in FORTRAN in the traditional, direct manner, it is simply

$$
\begin{array}{ll} 
& \text { SUM }=0 \\
& \text { DO } 11=1, N \\
& \text { SUM }=\operatorname{SUM}+A(1)
\end{array}
$$

The CFT generated scalar code, the results of which are given in Table VIII for various vector lengths. This algorithm was then rewritten in FORTRAN 10 in a fairly obscure manner so that CFT could vectorize most of the FORTRAN:

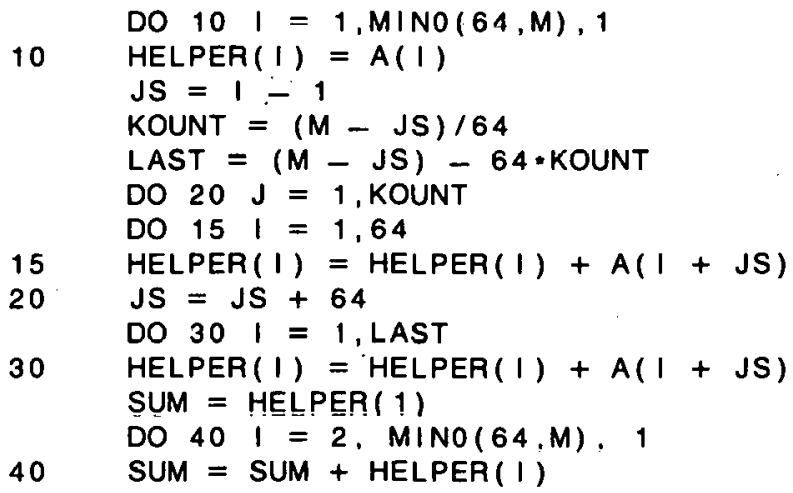

The CFT compiler generates vector code for loops 10, 15, and 30 . Table vIII shows the times and comparisons to the pure scalar version. The final version of this algorithm was written in CAL. By writing in CAL, we can calculate 64 partial sums in a vector register; then, using recursive characteristics of the vector functional unit, 11 we collapse the 64 partial sums to 8 partial sums. Finally, in a scalar loop, we can add the eight partial sums to obtain the single value SUM. As noted in Table VIIIb, as the vector lengths increase, the performance ratio of scalar to vector increases. This increase occurs because the scalar part of the vector calculation is done only once and hence can be amortized over all the vector elements. Even with the cost of subroutine linkage, the CAL sum routine has a break-even point at vector length of 3 . This routine is heavily used in the rewritten section of MD (Sec. III.D). 


\section{TABLE VI I I a}

SUM $=\mathrm{SUM}+\mathrm{A}(\mathrm{I})$

(a11 timings in microseconds)

\begin{tabular}{|c|c|c|c|}
\hline $\mathbf{M}$ & $\begin{array}{c}\text { Traditional } \\
\text { CFT }\end{array}$ & $\begin{array}{c}\text { Rewritten } \\
\text { CFT }\end{array}$ & CAL \\
\hline 1 & 3.2 & 6.4 & 4.0 \\
\hline 3 & 4.2 & 7.2 & 4.1 \\
\hline 10 & 7.6 & 10.8 & 4.1 \\
\hline 30 & 17.3 & 21.1 & 4.3 \\
\hline 100 & 51.5 & 40.1 & 5.3 \\
\hline 300 & 149.0 & 53.1 & 8.1 \\
\hline 1000 & 490.2 & 97.9 & 18.1 \\
\hline 3000 & 1465.2 & 225.2 & 46.6 \\
\hline
\end{tabular}

TABLE VIIIb

Comparison of Various Versions by Ratios

\begin{tabular}{|c|c|c|c|}
\hline $\mathbf{M}$ & Trad./Rewritten & Trad. /CAL & Rewritten/CAL \\
\hline 1 & $0: 5$ & 0.8 & 1.6 \\
\hline 3 & 0.6 & 1.0 & i. 8 \\
\hline 10 & 0.7 & 1.8 & 2.6 \\
\hline 30 & 0.8 & 4.0 & 4.9 \\
\hline 100 & 1.3 & 9.7 & 7.6 \\
\hline 300 & 2.8 & $\quad 18.4$ & 6.5 \\
\hline 1000 & 5.0 & 27.1 & 5.4 \\
\hline 3000 & 6.5 & 31.4 & 4.8 \\
\hline
\end{tabular}

The second example is also fairly simple:

$$
\begin{aligned}
& \text { DO } 1 I=1, N \\
& C(I)=1.0 \\
& I F(B(I) . E Q .0 .0) \text { GO TO } 1 \\
& C(1)=A(1) / B(1) \\
& 1 \text { CONTINUE }
\end{aligned}
$$

Written in the traditional FORTRAN way, it generates only scalar codes. By rewriting the FORTRAN statements using the utility procedure CVMGz (one of four procedures 10 that use the vector mask and merge hardware), we were able to remove the IF statement so that the FORTRAN could be vectorized by the compiler. The rewritten FORTRAN is:

$$
\begin{aligned}
& \text { DO } 11=1, N \\
& C(1)=C V M G Z(1.0, B(1), B(1)) \\
& C(1)=A(1) / C(1) \\
& C(1)=C V M G Z(1.0, C(1), B(1)) \\
& 1 \text { CONTINUE }
\end{aligned}
$$

Finally, we developed an efficiently written CAL subroutine that generated vector code similar to rewritten FORTRAN, by using the vector mask hardware. The CAL version also eliminated extra memory reference by better utilization of the vector registers. Table IX gives the times and ratios of the three versions. We note that for this particular example the rewritten FORTRAN is almost as good as the CAI.A. 
TABLE IXa

Example 2 ( $t$ ime in microseconds)

\begin{tabular}{|c|c|c|c|}
\hline M & $\begin{array}{l}\text { Traditional } \\
\text { CFT }\end{array}$ & $\begin{array}{l}\text { Rewritten } \\
\text { CFT }\end{array}$ & CAL \\
\hline 1 & 2.6 & 3.0 & 2.4 \\
\hline 3 & 5.3 & 3.0 & 2.5 \\
\hline 10 & 14.6 & 4.0 & 2.8 \\
\hline 30 & 40.6 & 7.2 & 4.4 \\
\hline 100 & 132.9 & 19.4 & 10.4 \\
\hline 300 & 398.2 & 53.6 & 27.8 \\
\hline 1000 & 1326.4 & 174.1 & 89.0 \\
\hline 3000 & 3979.5 & 517.9 & 263.4 \\
\hline
\end{tabular}

TABLE IXb

Example 2 (ratios)

\begin{tabular}{|c|c|c|c|}
\hline $\mathbf{M}$ & Trad./Rewritten & Trad./CAL & Rewritten/CAL \\
\hline 1 & 0.87 & 1.10 & 1.26 \\
\hline 3 & 1.70 & 2.09 & 1.22 \\
\hline 10 & 3.68 & 5.17 & 1.40 \\
\hline 30 & 5.64 & 9.23 & 1.63 \\
\hline 100 & 6.87 & 12.73 & 1.85 \\
\hline 300 & 7.43 & 14.32 & 1.92 \\
\hline 1000 & 7.62 & 14.90 & 1.95 \\
\hline 3000 & $7: 68$ & 15.11 & 1.97 \\
\hline
\end{tabular}

The third example involves the PIC routine PARMOVE discussed in Sec. III. This routine is a good test for vector machines because it has several Do loops which cannot be vectorized for a vector machine or executed in parallel on a parallel computer. The structure of one such loop. called gather-scatter, is detailed below:

Given a random set of indices $I(J)$, then

$$
\begin{array}{ll} 
& D O 1 J=1, N \\
I J=I(J) \\
1
\end{array}
$$

Such scalar calculations exist in the majority of production programs and can significantly affect the overall performance of vector or parallel machines. 12

Usually, the programmer resorts to dimensioning all temporary variables on the left-hand side of the equal sign. This task significantly increases the storage requirements to take advantage of the vector hardware.11 To avoid this problem, we rewrote PARMOVE in CAL, eliminating all but five 64-word arrays needed to hold temporary variables. Our efforts resulted in approximately a fourfold improvement in performance time (down to 1.7 microseconds per particle) over the CRAY rewritten version and a dramatic reduction in space needed (see Table X). 
TABLE X

Size of PARMOVE (in decimal-words)

Tradit Iona1

CFT

168
Rewritten
CFT

2392
CAL

480

The University of Michigan (UM) designed and developed a CRAY-1 cross assembler ${ }^{3}$ and simulator ${ }^{14}$ that executes on their Amdahl computer under the Michigan Timesharing System. During a workshop on parallel processing at UM, we had occasion to exercise the simulator on the CAL version of PARMOVE. One of the results the simulator provides is the MFLOP rate of that routine. PARMOVE performed at a rate of 40 MFLOPS, including the scalar loops that were in PARMOVE to perform the gather-scatter.

Finally, we ran some tests usiny the VFFA and VFFs routines. Like PARMOVE, these routines can be vectorized by the CFT compiler, provided that all scalar temporary variables are dimensioned; dimensioning requires the use of more memory. The assembly language version of the two routines, on the other hand, not only executes faster but requires no extra memory and is, in fact, smaller than the scalar version of the codes. Table XI gives the times for various versions and various mesh sizes.

The two routines were also executed on the simulator at the University of Michigan. VFFA executes at 89 MFLOPS and VFFS at 103 MFLOPS.

TABLE XI

Fourier Anulysis Routines VFFA and VFFS ( $t$ imes in $\mathrm{ms}$ )

\begin{tabular}{|c|c|c|c|c|}
\hline & $32 \times 32$ & $64 \times 64$ & $128 \times 128$ & $256 \times 256$ \\
\hline Traditional & 4.48 & 21.44 & 100.97 & 467.10 \\
\hline Rewritten & 1.03 & 3.31 & 15.01 & 68.00 \\
\hline CAL & 0.29 & 1.24 & 6.06 & 28.22 \\
\hline
\end{tabular}

Both these routines and PARMOVE will be used under a production environment for the ionion plasma simulation in an electric quadrupole mass filter. 


\section{MACHINE ENVIRONMENT STUDIES}

An environmental inquiry program MACHAR was run by $W$. $J$. Cody of the Applied Mathematical Sciences Section of AMD to determine the basic parameters of the CRAY-1 floating-point arithmetic. The first version of MACHAR uncovered a number of discrepancies between the behavior of the floating-point arithmetic unit and the CRAY-1 machine documentation.

In particular, it is possible to generate floating-point numbers that are too small, according to the documentation, but that do not underflow. In addition, overflow may occur one exponent digit too soon. Apparently the CRAY-1 hardware checks for an out-of-range exponent in the result of a multiplication operation before the postnormalization shift and corresponding exponent adjustment. This check fails to detect "underflow" in certain cases and prematurely indicates overflow in others. As a result of these findings MACHAR was modified so that it now runs correctly on the CRAY-1.

Using the revised version of MACHAR, Cody then tested the following singleprecision function programs with scalar (but not vector) arguments: ALOG/ALOG10, ATAN/ATAN2, EXP, SIN/COS, SQRT, and TANH. In several cases the tests did not run to completion because the acceptable argument range for the function program (in particular, for EXP) was smaller than necessary and out-of-range arguments caused job termination.

Annotated output from MACHAR (both single- and double-precision versions) and results from the function tests are given in Appendix $A$. The test results are indicative, but not definitive, of places where the function programs are inferior to those available on other machines. 


\section{I/O TESTS}

A major problem that has always confronted large-scale scientific computers with fast CPU's is the reading and writing of large amounts of data. These data must be moved between the main memory and the secondary storage units (disks), while the CPU performs meaningful work. To date, disk technology remains limited by the rotational speed of the disk platters, i.e., the mechanical parts of the disk units. Thus, even on the CRAY-1, the theoretical transfer rate of 502,691 words per second is only slightly faster than that of the CDC 7600 disks.

The only way the CRAY CPU can be matched to the disks is by having several disks transferring data in parallel, each on separate input/output ( $1 / 0$ ) channels. The CRAY-1 has 12 independent $I / 0$ channels; currently, NCAR users have access to four of these, connected to four independent disk units. In addition to the hardware needed to handle high-speed I/O, we must consider the operating system on the CRAY-1. The CRAY-1 Operating System (COS) is a multiprogramming operating system; that is, most of the time several user jobs reside in memory at a time, and the system attempts to provide for efficient use of the available resources. To manage large sequential data files and to keep the CPU busy, a triple buffer technique has been devised, whereby two disk units input data and two output data, while the CPU carries out the user's calculations.

To evaluate this technique, we used an I/O test, developed at LASL, that measures several important quantities:

1) the transfer rate of the disk for various-sized buffers,

2) the amount of CPU time needed by the system to perform the I/O, and

3) the effects of multiple channels.

The test has been set up to run where a single user has access to the whole machine, since it is important that no other user program affects the position of the disk heads. Furthermore, to ensure a proper measure of the buffer transfer rate, the test does not use any system buffers. Data are transferred directly into the user field length where he allocates the appropriate buffer space. Cos permits the user to manage his $I / O$ in just such a manner.

The results are shown in Table XII. The first column denotes the buffer space in the user fleld length in tracks (9216 64-bit words per track). The second column indicates the number of different channels being used. The third column indicates the transfer rate per channel in words per second. Finally, the last column indicates the percentage of the CPU needed to perform the I/O.

It should be noted that a channel on the CRAY-1 has a 1048-word buffer. After a section of data has been moved to this buffer, an interrupt is issued by the channel that must be serviced by the operating system. It is this action that requires the use of the CPU performing $I / O$.

TABLE XII

CRAY-1 I/0 Test Results

\begin{tabular}{|c|c|c|c|}
\hline Tracks & Channels & Rate/Channel & Percentage CPU for $1 / 0$ \\
\hline 1 & 2 & 460800 & 13.9 \\
\hline 1 & 4 & 425354 & 27.2 \\
\hline 2 & 2 & 460800 & 11.5 \\
\hline 2 & 4 & 460800 & 19.5 \\
\hline 5 & 2 & 4 4ตกรกก & 10.2 \\
\hline 5 & 4 & 460800 & 10.5 \\
\hline
\end{tabular}




\section{DISCUSSION}

Table XIII summarizes the results of our performance comparisons. These data are, of course, preliminary and deal with an extremely small set of codes. It should also be noted that running times will vary considerably, depending upon the individual routine and programming techniques. Nevertheless, some comments can be made.

TABLE XIII

Improvement Factor on the CRAY-1 over the IBM $370 / 195$

with Various Versions of Code

\section{Direct Trans. Minor Code Extensive Code Changes Changes}

$\begin{array}{lccc}\text { Ptolemy } & 1.4 & 2.0 & 10.0 \\ \text { Dirac-Slater } & 2.3 & 2.6 & - \\ \text { PIC } & 3.4 & 7.6 & 22.0 \\ \text { PARMOVE } & 2.4 & 5.7 & 24.5 \\ \text { VFFA + VFFS } & 1.8 & 7.7 & 37.9 \\ \text { MD } & 1.3 & 1.7 & - \\ \text { ICEPEL } & 1.7 & 2.2 & - \\ \text { JWMBENCH } & 1.0 & 1.3 & 4.5 \\ \text { DIF3D } & 1.0 & 1.3 & - \\ \text { TSHTR } & 2.0 & - & - \\ \text { ALPHFN * } & 30.0 & - & -\end{array}$

\footnotetext{
-known to be efficiently written for the IBM $370 / 195$

- set of new routines written in FORTRAN, such that CFT generates efficient vector instructions
}

Experience indicates that direct translation from the IBM $370 / 195$ to the CRAY-1 can result in up to a twofold improvement in execution time (see column 1 of Table XIII). Moreover, the CRAY-1 execution times should continue to decrease as the CFT compiler matures. As Section IV illustrates, we are a long way from exploiting the full potential of the CRAY-1.

The improvement realized from direct translation. while in itself not much greater than one would hope for in utilizing an advanced computer, nevertheless is significant in that all of the test programs were designed and developed on the traditional sequential computers and most of the code reflects this fact. Thus, we may expect to encounter greater improvements in execution time as these programs are modified to expose the parallel and vector structure for the compiler to recognize and exploit.

Until the maturity of the CFT compiler is equivalent to that of the IBM FORTRAN H extended opt 2 or CDC FTN opt 2 , code changes such as eliminating an unnecessary overflow test or dimensioning scalar temporary variables inside a Do loop will be needed to further reduce the execution time on the CRAY-1. As shown in the second column of Table XIII. such changes can improve run time from twoto seven-fold.

Finally, to obtain the most dramatic improvements, extensive code rewriting will be necessary. In all the cases where we undertook such rewriting, execution times decreased significantly, in three cases by a factor of more than 20 (see cols. 1 and 3 of Table XIII). Here, of course, the user must carefully examine the structure of his code, identify areas where vector operations can replace 
scalar techniques, and design new and efficient vector implementations for such areas. In most cases, these changes involve only a superficial modification of the FORTRAN. Occasionally, however, we find a program for which no efficient parallel implementations are available, no matter what changes we attempt to make at the FORTRAN level. Thus, we must examine the algor 1 thm that the program is using and determine whether another algorithm can be used that is more conducive to vector and parallel computer architecture. To change algorithms is a major effort, even for the individual who knows and understands the program, but the payoff for such changes is usually significant. We hope to undertake such an effort, as part of a future project, starting with the large DIF3D program discussed in Sec. III.E.

We recognize that the ordinary user cannot be expected to make the extensive changes to his program that have been discussed in this section; for the more sophisticated programmer, however, the investment of effort is worth the improvement in program running time. For example, one man-day spent tuning the 5000-line section of Ptolemy achieved a tenfold improvement in execution speed. 


\section{SUMMARY AND CONCLUSIONS}

The growing complexity of research in the physical, biomedical, and environmental sciences appears in some cases to require sophisticated calculations that are beyond both the capacity and the capability of Argonne's Central Computing Facility. A primary objective of the Advanced Scientific Computers Project was to determine the potential effect of class VI computers on the Argonne workload. We have demonstrated that the CRAY-1 can be used by some Argonne researchers not only to achieve faster execution times but also to solve problems that would otherwise be prohibitive on any traditional computer.

The impact on Argonne from the lack of permanent access to a machine such as a CRAY-1 includes:

1) the inability to solve certain interesting complex problems that are state-of-the-art in the various scientific technologies, and

2) the inability to remain competitive in certain areas with other national laboratories that already have CRAY's (e.g., LASL, LLL, NCAR, and NMFECC) and arc able to pursue the large-scale modeling that requires such computational capabilities.

These limitations can substantially affect the funding of major DoE projects at Argonne. Thus we conclude that if argonne is to maintain its leadership in scientific investigations, it should offer state-of-the-art computing capabilities.

We also note that, although supercomputers have generally been considered "software poor," users involved in the Advanced Scientific Computers Project have been encouraged by the ability to convert significant portions of production codes and to execute them correctly on the CRAY-1 with relative ease. Undoubtedly, as the CRAY software grows in sophistication, the effort required to develop or translate code will decrease, while the performance realized will increase. Indeed, test runs made since the data were collccted for this report indicate that better instruction scheduling in the CFT compiler can reduce run times by 10 to 30 percent.

Clearly, the CRAY software and hardware are capable of handing the complex calculations required for advanced scientific research. Moreover, CRAY Research has indicated that a link between the CRAY-1 and computers such as the IBM $370 / 195$ will be available by April 1979. Such a link would considerably facilitate integration of the vector machine into Argonne's Central Computing Facility. 


\section{REFERENCES}

1. R. L. Patrick, ANL Computer Requirements, July 1977

2. F. H. Harlow, The Particle-in-cell Computing Method for Fluid Dynamics, Methods of Comp. Physics, 3, 319-343 (1964)

3. R. L. Morse, Multidimensional Plasma Simulation by the Particle-in-cell Method, Methods of Comp. Physics, 9, 213 (1970)

4. D. W. Forslund, C. W. Nielson, and L. E. Rudsinski, Vectorized PIC Simulation Codes on the CRAY-1, 1978 LASL Workshop on Vector and Parallel Processors, Los Alamos, New Mexico, September 20-22, 1978

5. G. D. Bergland, A Fast Fourier Transform Algorithm for Real-Valued Series, Communications of the ACM, 11, 703-710 (1968)

6. B. Buzbee, F. Dorr, J. George, and G. Golub, The Direct Method for Solving Poisson's Equation, SIAM J. of Num. Anal., 7, 677 (1970)

7. B. Buzbee, F. Dorr, J. George, and G. Golub, Solutions of the Discrete Poisson Equation on Irregular Regions, SIAM J. on Num. Anal., 8, 722 (1971)

8. D. R. Ferguson and K. L. Derstine, Optimized Iteration Strategies and Data Management Considerations for Fast Reactor Finite Difference Diffusion Theory Codes, Nuc. Sci. \& Eng., 64, 593-604 (1977)

9. J. J. Dongarra, J. R. Bunch, C. B. Moler, and G. W. Stewart, LINPACK User's Guide, Philadelphia: SIAM, to appear. See also J. J. Dongarra, LINPACK working Note No. 11: Some LINPACK Timings on the CRAY-1, LOS Alamos Scientific Laboratory Informal Report LA-7389-MS, August 1978

10. CRAY-1 FORTRAN (CFT) Reference Manual, No. 2240009, Cray Research, Inc., 1977

11. CRAY-1 Computer System Hardware Reference Manual, No. 2240004, Cray Research, Inc., 1977

12. L. Rudsinski and J. Worlton, The Impact of Scalar Performance on Vector and Parallel Processors, High-speed Computers and Algorithm Organization, ed. David J. Kuck, Duncan Laurie, and Ahmed H. Sameh (New York: Academic Press, Inc., 1977), p. 451

13. W. G. Ames, A CRAY-1 Cross Assembler, University of Michigan SEL Report No. 120 , September 1978

14. D. A. Orbits, $A$ CRAY-1 Simulator, University of Michigan SEL Report No. 118, September 1978 


\section{ACKNOWLEDGMENTS}

We are indebted to Jim cody (Applied Mathematics Division) for the information in Section $V$ and Appendix $A$ and to Clair Nielsen (L-Division, Los Alamos Scientific Laboratory) for the vectorized version of the PIC program, for the program used to perform the I/O tests, and for many discussions. Most of the documentation and data for section III were provided by the following people: Keith Derstine (Applied Physics), Floyd Dunn (Reactor Analysis and Safety), Dale Koelling (Solid State Sciences), M. A. Moneim (Reactor Analysis and Safety), Steven Pieper (Physics), Aneesur Rahman (Solid State Sciences), Roshan Rivetna (High Energy Physics), and Al Wagner (Chemistry). George Whittington provided useful editorial assistance for this report. We extend special thanks to paul Messina for his suggestions and comments. 


\section{APPENDIX A \\ Testing the CRAY-1 Parameters and Elementary Function Subroutines}

\section{SUBROUTINE MACHAR}

C

CD

CD

C

C

C

C

$\mathrm{C}$

C

C

C

C

C

C

C

C

C

C

C

C

C

C

C

C

C

C

C

C

C

C

C

C

C

C

C

C

C

C

C

SUBROUT INE MACHAR( I BETA, I T, I RND, NGRD, MACHEP, NEGEP, I EXP, MINEXP, 1 MAXEXP , EPS , EPSNEG, XMIN, XMAX)

\footnotetext{
INTEGER I , I BETA, IEXP, IRND, I T, I Z, J, K, MACHEP, MAXEXP, MINEXP ,
} $1 \quad M X, N E G E P, N G R D$

REAL A, B, BETA, BETAIN, BETAM 1, EPS, EPSNEG, ONE, XMAX, XMIN, Y, Z, ZERO DOUBLE PRECISION A, B, BETA, BETAIN, BETAM1, EPS, EPSNEG, ONE, XMAX, 1 $X M I N, Y, Z, Z E \dot{R} O$

THIS SUBROUTINE IS INTENDED TO DETERMINE THE CHARACTERISTICS OF THE FLOATING-POINT ARITHMETIC SYSTEM THAT ARE SPECIFIED BELOW. THE FIRST THREE ARE DETERMINED ACCORDING TO AN ALGORITHM DUE TO M. MALCOLM, CACM 15 (1972), PP. 949-951, INCORPORATING SOME, BUT NOT ALL, OF THE IMPROVEMENTS SUGGESTED BY M. GENTLEMAN AND S. MAROVICH, CACM 17 (1974), PP. 276-277. THE VERSION GIVEN HERE IS FOR SINGLE PRECISION. CARDS CONTAINING CD IN COLUMNS 1 AND 2 CAN BE USED TO CONVERT THE SUBROUTINE TO DOUBLE PRECISION BY REPLACING EXISTING CARDS IN THE OBVIOUS MANNER.

LATEST REVISION - MAY 16, 1978.

AUTHOOR - W. J. CODY ARGONNE NATIONAL LABORATORY

IBETA IS THE RADIX OF THE FLOATING-POINT REPRESENTATION

IT IS THE NUMBER OF BASE IBETA DIGITS IN THE FLOATING-POINT SIGNIF I CAND

IRND $\quad=0$ IF THE ARITHMETIC CHOPS,

NGRD $=0$ IF IRND=1, OR IF IRND=0 AND ONLY IT BASE IBETA DIGITS HAHIICIHAIE IN THE POST NORMALIZATION SHIFT OF THE FLOATING-POINT SIGNIFICAND IN MULTIPLICATION

1 IF IRND=0 AND MORE THAN IT BASE IBETA DIGITS PARTICIPATE IN THE POST NORMALIZATION SHIFT OF THE FLOATING-POINT SIGNIFICAND IN MULTIPLICATION

MACHEP IS THE EXPONENT ON THE SMALLEST POSITIVE FLOATING_POINT NUMBER EPS SUCH THAT 1.0+EPS.NE. 1.0

NEGEPS IS THE EXPONENT ON THE SMALLEST POSITIVE FL. PT. NO. NEGEPS SUCH THAT 1.0-NEGEPS .NE. 1.0, EXCEPT THAT NEGEPS IS BOUNDED BELOW BY IT-3

IEXP IS THE NUMBER OF BITS (DECIMAL PLACES IF IBETA = 10) RESERVED FOR THE REPRESENTATION OF THE EXPONENT OF A FLOATING-POINT NUMBER

MINEXP IS THE EXPONENT OF THE SMALLEST POSITIVE FL. PT. NO. $X M I N$

MAXEXP IS THE EXPONENT OF THE LARGEST FINITE FLOATING-POINT NUMBER XMAX

EPS

IS THE SMALLEST POSITIVE FLOATING-POINT NUMBER SUCH THAT 1.0 HEPS.NE. 1.0. IN PARTICULAR, EPS = IBE IA* MACHEP

EPSNEG IS THE SMALLEST POSITIVE FLOATING-POINT NUMBER SUCH THAT 1.0-EPS .NE. 1.0 (EXCEPT THAT THE EXPONENT 


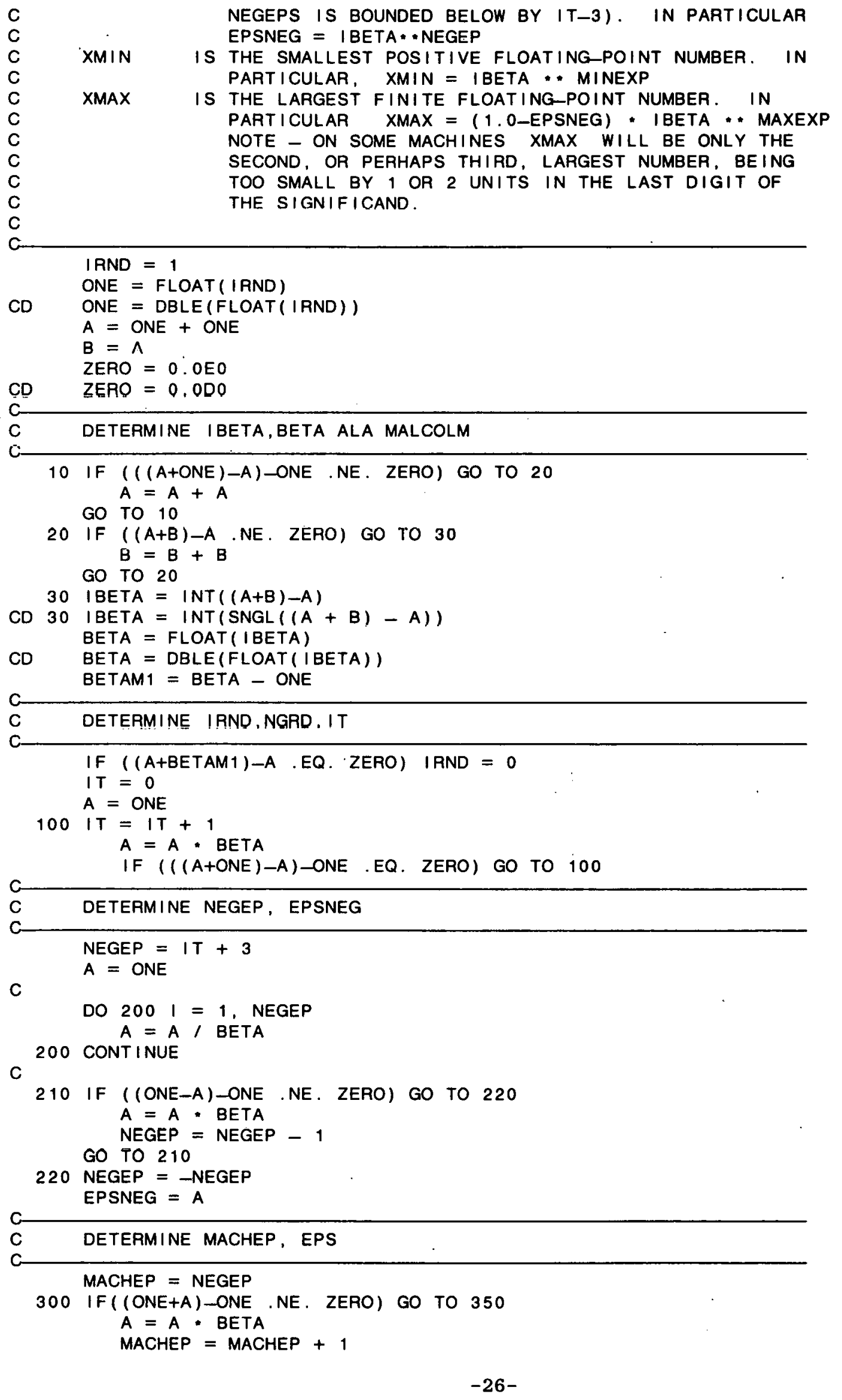




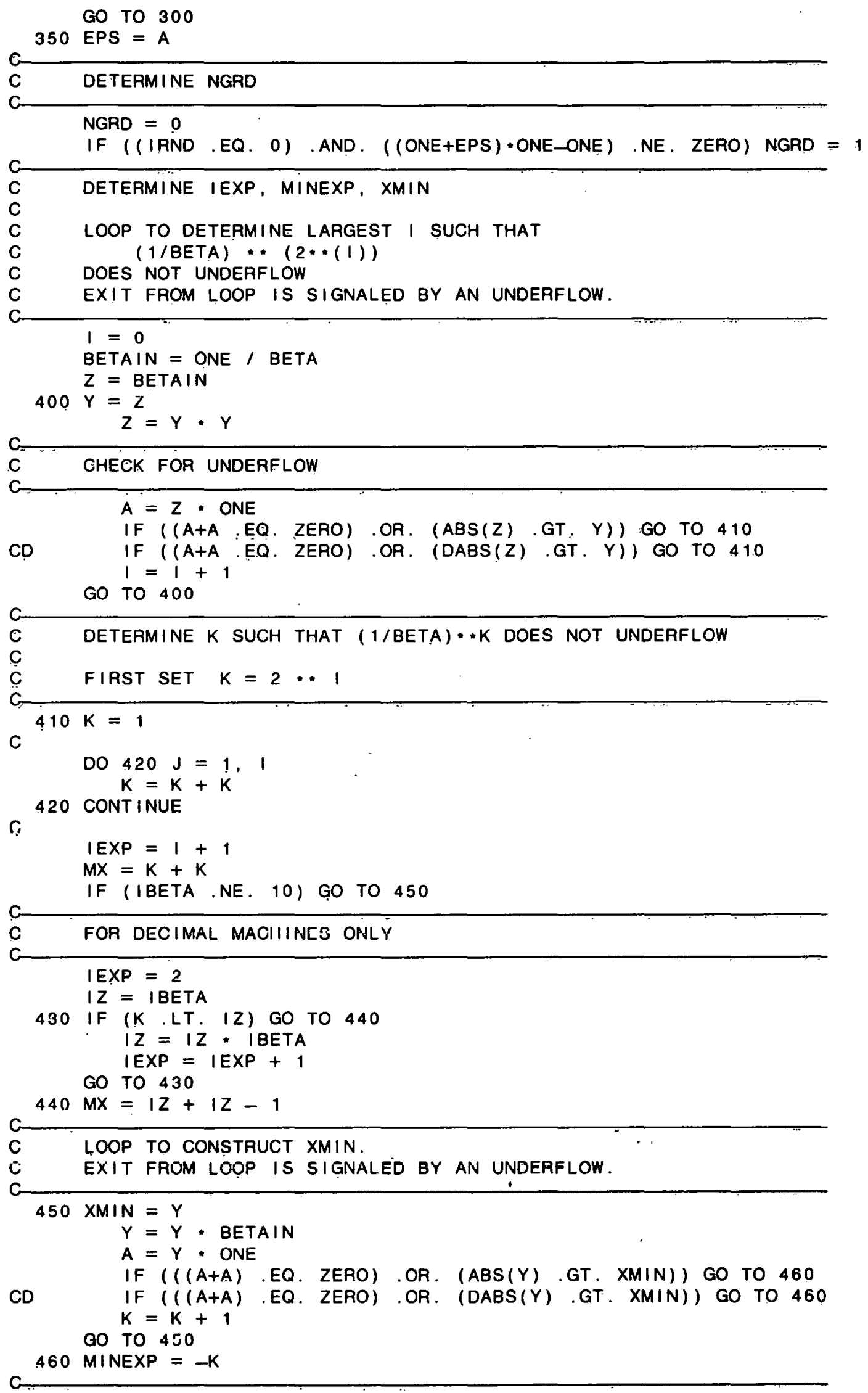




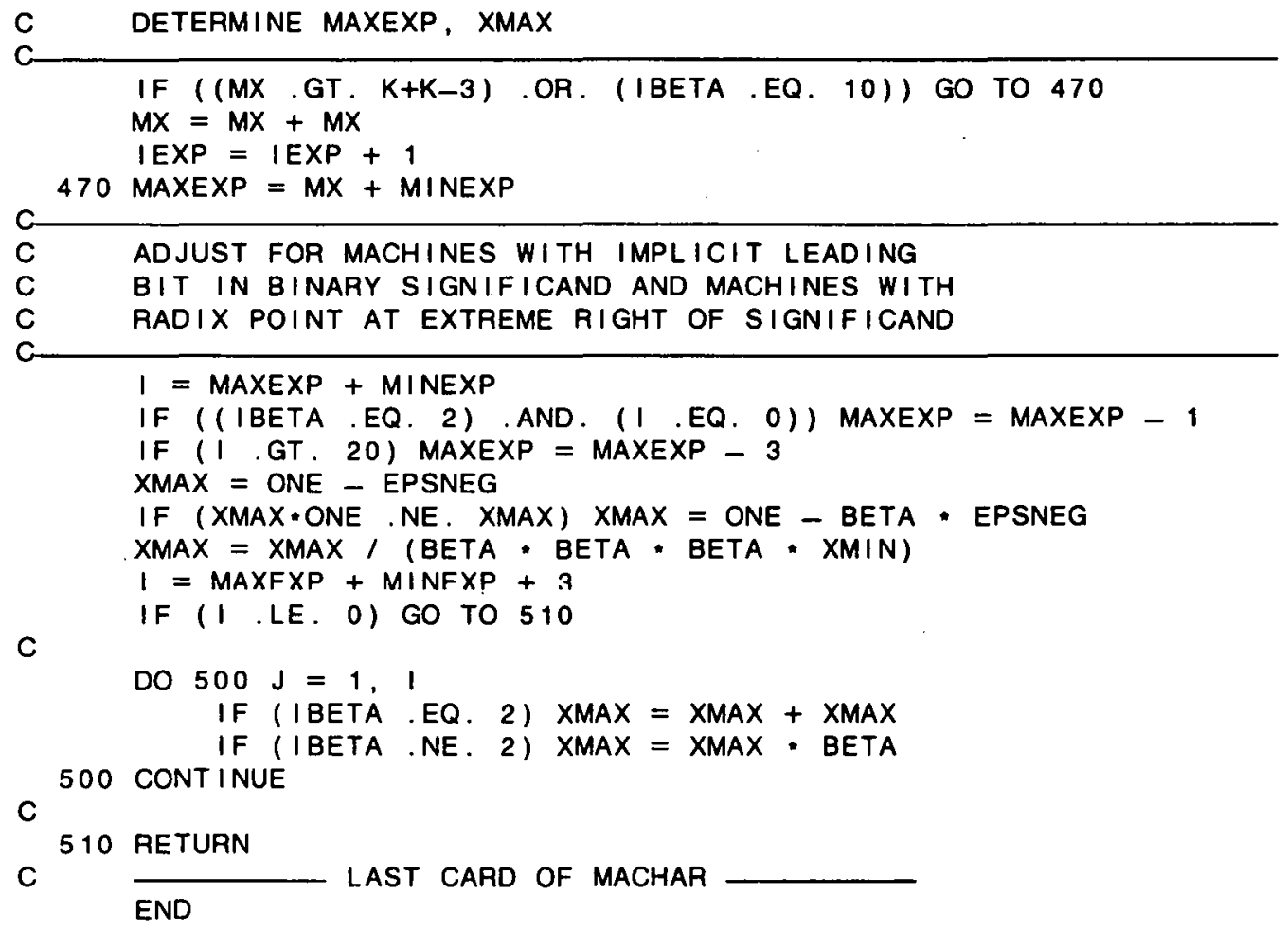




\section{I OUTPUT FROM MACHAR}

\section{A. Single-precision MACHAR (revised 5/15/78)}

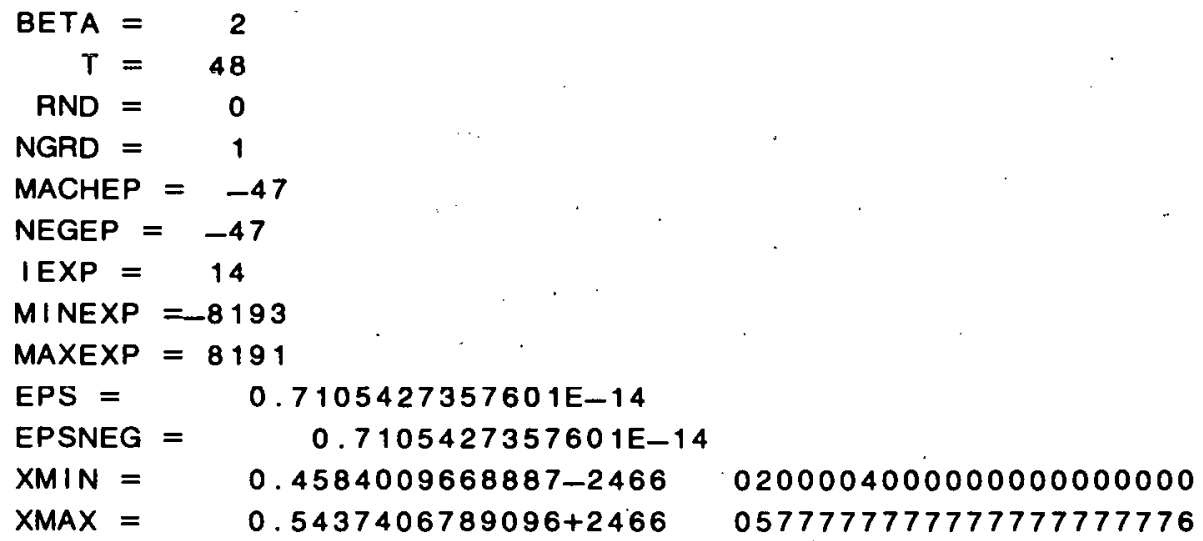

\section{Operation}

Decimal

Octal

$X=X M I N * 0.5 E 0$

$X * 1.0 E 0$

$x \cdot 2$. OEO

$\mathrm{X}+\mathrm{X}$

$x=2$. OEO $-x$

$(x+x)-x$

$x+0.0 E 0$

2. $0 \cdot x+x$

$0.0+x$

$x-0.0 E 0$

$X+0.0 E 0$
$0.0000000000000 E+00$

0. $0000000000000 E+00$

$0.4584009668887-2466$

$0.0000000000000 E+00$

$0.0000000000000 E+00$

0. $0000000000000 E+00$

$0.0000000000000 E+00$

$0.6876014503331-2466$

$0.0000000000000 E+00$

$0.0000000000000 E+00$

$0.0000000000000 E+00$
0177774000000000000000 0200004000000000000000 0000000000000000000000 0177774000000000000000 0000000000000000000000 0177774000000000000000 0200006000000000000000 0177774000000000000000 0177774000000000000000 0177774000000000000000

B. Double-precision MACHAR (revised $8 / 17 / 78$ )

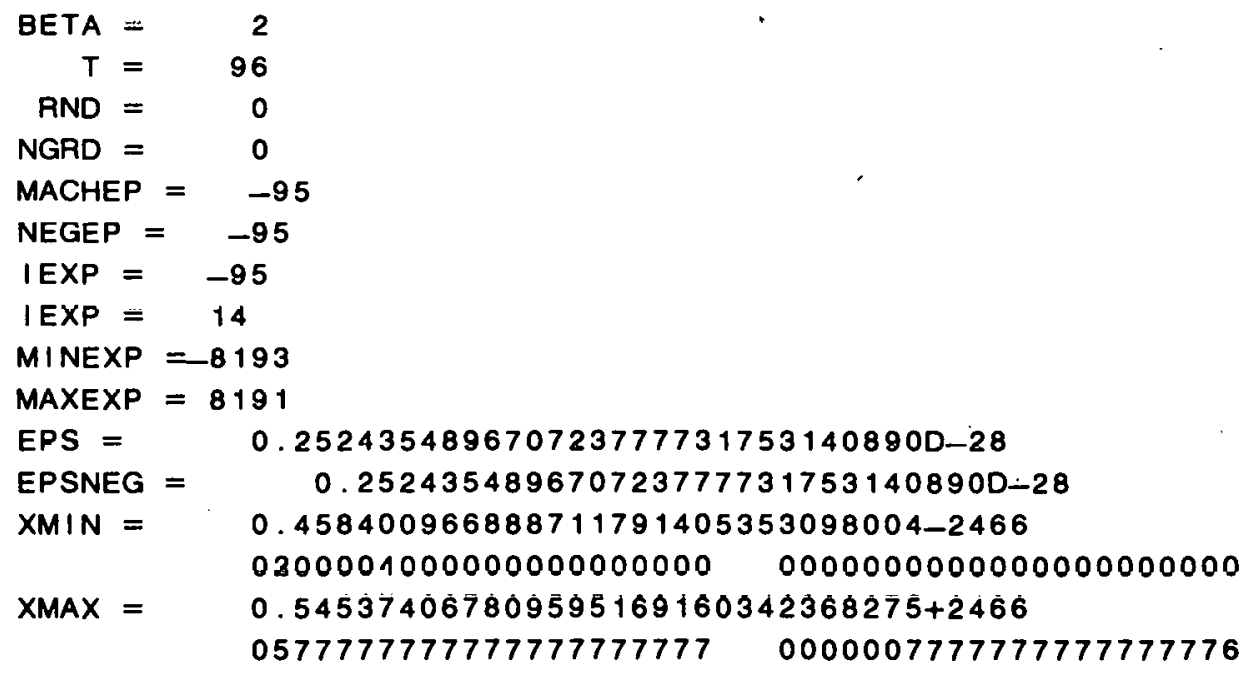


III. FUNCTION TEST RESULTS

CFT Mathemat1cal Subprogram Library

\begin{tabular}{|c|c|c|c|c|c|}
\hline Function & Interval (a) & Test & $\underline{M E}(\underline{b})$ & $\underline{R M E}(c)$ & Commer \\
\hline ALOG & $(1-2-16,1+2-16)$ & 1 & $2 *-45.61$ & $2 *-47.24$ & a \\
\hline ALOG & $(\sqrt{.5}, .9375)$ & 2 & $2 *-45.77$ & $2 *-47.67$ & a \\
\hline ALOG & $(16,240)$ & 3 & $2 * *-46 \cdot 12$ & $2 * *-47.69$ & c \\
\hline ALOG 10 & $(\sqrt{.1}, .9)$ & 4 & $2 *-44.57$ & $2 \cdot-46.64$ & b \\
\hline ATAN & $(-.0625, .0625)$ & 1 & $2 *-47.00$ & $2 *-48.00$ & a, c \\
\hline ATAN & $(.0625,2-\sqrt{3})$ & 5 & $2 *-46.35$ & $2 *-47.86$ & a. c \\
\hline ATAN & $(2-\sqrt{3}, \sqrt{2}-1)$ & 6 & $2 * *-45.61$ & $2 *-47.33$ & $\mathbf{a}, \mathbf{c}$ \\
\hline ATAN & $(\sqrt{2}-1,1.0)$ & 6 & $2 *-45.45$ & $2 *-46.95$ & a, c \\
\hline EXP & $(-.2841, .3466)$ & 7 & $2 * *-44.69$ & $2 *-46.60$ & $d$ \\
\hline EXR & $(3.166,6611.0)$ & 8 & $2:-448 h$ & $?:-4 h$ f? & d \\
\hline EXP & $(6.931,2800.0)$ & 8 & $2 *-44.95$ & $2 *-46.66$ & d, $\theta$ \\
\hline SIN & $(0, \pi / 2)$ & 9 & $2 \cdot \div-45 \cdot 16$ & $2 *-46.56$ & $\mathbf{a}$ \\
\hline$S I N$ & $(6 \pi, 6.5 \pi)$ & 9 & $2 * *-32.26$ & $2 *-37.50$ & $f$ \\
\hline $\cos$ & $(7 \pi, \quad 7.5 \pi)$ & 10 & $2 \cdot-30.67$ & $2 *-36.12$ & f. $g$ \\
\hline SQRT & $(\sqrt{.5}, 1.0)$ & 11 & $2 *-47.50$ & $2 *-46.65$ & h \\
\hline SQRT & $(1, \sqrt{2})$ & 11 & $2 *-47.00$ & $2 *-48 \cdot 30$ & $n$ \\
\hline TANH & $(.125, .5493)$ & 12 & $2 *-43.47$ & $2 * *-45.47$ & b \\
\hline TANH & $(.6743,17.68)$ & 12 & $2 *-45.85$ & $2 * *-47.31$ & c \\
\hline
\end{tabular}

(a) Based on 2000 random arguments

(b) Maximum Relative Error; basic roundoff is $2 *-48$

(c) Root Mean Square Relative Error

\section{Test}

1) Comparison against Taylor series

2) Check identity

$$
\operatorname{Ln}(x)=\operatorname{Ln}(17 x / 16)-\operatorname{Ln}(17 / 16)
$$

3) Check identity

$$
\operatorname{Ln}\left(x^{2}\right)=2 \operatorname{Lin}(x)
$$

4) Check identity

$$
\log (x)=\log (11 x / 10)-\log (11 / 10)
$$

5) Check identity $\tan ^{-1}(x)=\tan ^{-1}(1 / 16)+\tan ^{-1}[(x-1 / 16) /(1+x / 16)]$

6) Check identity

$$
2 \tan ^{-1}(x)=\tan ^{-1}\left[2 x /\left(1-x^{2}\right)\right]
$$

7) Check identity

$$
\exp (x-1 / 16)=\exp (x) / \exp (1 / 16)
$$

8) Check identity $\exp (x-2.8125)=\exp (x) / \exp (2.8125)$

9) Check identity

$$
\sin (x)=3 \sin (x / 3)-4 \sin ^{3}(x / 3)
$$

10) Check identity

$$
\cos (x)=4 \cos ^{3}(x / 3)-3 \cos (x / 3)
$$

11) Check identity

$$
x=\operatorname{sqrt}\left(x^{2}\right)
$$


12) Check identity

$$
\tanh (x)=\frac{\tanh (x-1 / 8)+\tanh (1 / 8)}{1+\tanh (x-1 / 8) \tanh (1 / 8)}
$$

\section{Comment}
a) Error larger than expected.
b) Error too large
c) Error acceptable
d) Errors larger than expected by about 1 bit
e) Argument range not in agreement with documentation
f) Bad argument reduction
g) Probably poor linkage to SIN routine
h) Excellent 
Internal:

L. Rudsinski (87)

A. B. Krisciunas

ANL Contract File

ANL Libraries (5)

TIS Files (6)

\section{External:}

DOE-TIC, for distribution per UC-32 (205)

Manager, Chicago Operations Office

Chief, Office of Patent Counsel, $\mathrm{CH}$

President, Argonne Universities Association

Applied Mathematics Division Review Committee:

P. J. Eberlein, SUNY at Butfalo

G. Estrin, U. California, Los Angeles

W. M. Gentleman, II. Waterinn

J. M. Ortega, North Carolina State U.

E. N. Pinson, Bell Telephone Labs.

S. Rosen, Purdue U.

D. M. Young, Jr., U. Texas at Austin 